\title{
Parameter Estimation Techniques for a Class of Nonlinear Hysteresis Models
}

\author{
Ralph C. Smith and Andrew G. Hatch \\ Center for Research in Scientific Computation \\ Department of Mathematics \\ North Carolina State University \\ Raleigh, NC 27695-8205 \\ rsmith@eos.ncsu.edu, aghatch@eos.ncsu.edu
}

\begin{abstract}
This paper addresses the development of parameter estimation techniques for a class of models used to characterize hysteresis and constitutive nonlinearities inherent to ferroelectric, ferromagnetic and ferroelastic materials employed in a wide range of actuators and sensors. These models are formulated as integral equations with known kernels and unknown densities to be identified through least squares techniques. Due to the compactness of the integral operators, the resulting discretized models inherit ill-posedness which must be accommodated through regularization. The accuracy of regularized finite-dimensional models is illustrated through comparison with experimental data.
\end{abstract}




\section{Introduction}

Hysteresis and constitutive nonlinearities are inherent properties of a wide range of piezoceramic (PZT), magnetic, and shape memory alloy (SMA) compounds being considered as actuators and sensors in aeronautic, aerospace, automotive, industrial and biomedical applications. In some regimes, hysteresis can be mitigated through restricted input levels, amplifier designs, or feedback mechanisms. In general, however, these properties are ubiquitous and are often inexorably related to material attributes which provide the materials with unique transducer capabilities. For example, damping provided by a shape memory tendon is proportional to the area of the hysteresis loop. Hence optimal tendon design to attenuate wind or earthquake-induced vibrations in civil structures requires maximal hysteresis rather than operation in approximately linear regimes. This necessitates the development of models, parameter estimation techniques, and model-based control designs which incorporate the nonlinear hysteresis mechanisms in a manner which facilitates subsequent implementation.

A number of modeling strategies have been proposed but three stand out in the sense that they provide unified frameworks for characterizing hysteresis in ferroelectric, ferromagnetic and ferroelastic materials, which are collectively referred to as ferroic compounds. These three approaches are homogenized energy models $[8,10,18-20,24,25]$, Preisach formulations $[2,3,5,7,16,26]$, and domain wall models $[6,8,14,21,22]$.

As detailed in [17], the domain wall framework is efficient to implement but requires a priori knowledge of turning points to guarantee closure of biased minor loops. This precludes its use in certain feedback designs where turning points are determined by measured or estimated states of the system. The classical Preisach framework requires the properties of congruency and deletion [11,12] which are overly restrictive for a number of materials and applications. As detailed in [1], these restrictions have been eliminated for magnetic material characterization through the development of extended Preisach models; however, these extensions come at the price of increased complexity, and the maturity of extended Preisach models for PZT and SMA lags far behind the magnetic theory. The homogenized energy framework is the most recent of the three and is based on a combination of energy analysis at the lattice level and stochastic homogenization techniques to construct macroscopic models. Due to its energy basis, it incorporates certain frequency, temperature, and stress dependencies which makes it applicable for a wide range of transducer designs and operating regimes. Furthermore, it is illustrated in [23] that this framework provides an energy basis for certain extended Preisach formulations. Due to its flexibility and generality, we employ the homogenized energy framework in this paper and construct parameter estimation techniques in this context.

In Section 2, we summarize the homogenized energy model for ferroelectric (e.g., PZT), ferromagnetic (e.g., iron, Terfenol-D), and ferroelastic (e.g., SMA) compounds to illustrate its structure and generality. Specifically, it will be noted that the models are formulated as integral equations with known kernels and unknown densities. The compactness of the integral operator is established in Section 3 and, in Section 4, it is shown that this leads to ill-posedness in the inverse problem associated with estimating the densities given a set of measured input-output data. Experimental validation results are summarized in Section 5. To simplify the discussion, Sections 3 through 5 will focus on the polarization model. Due to the general nature of the framework, however, the results are equally applicable to the magnetic and shape memory alloy models. 


\section{$2 \quad$ Free Energy Framework}

We summarize in this section the free energy framework for characterizing hysteresis in ferroelectric, ferromagnetic and ferroelastic materials. Details regarding the development and validation of these models can be found in $[10,19,20,25]$.

\subsection{Polarization Model}

\subsubsection{Local Polarization Model}

To quantify the internal and electrostatic energy at the lattice level, we employ the Helmholtz energy

$$
\psi(P)= \begin{cases}\frac{1}{2} \eta\left(P+P_{R}\right)^{2} & , P \leq-P_{I} \\ \frac{1}{2} \eta\left(P-P_{R}\right)^{2} & , P \geq P_{I} \\ \frac{1}{2} \eta\left(P_{I}-P_{R}\right)\left(\frac{P^{2}}{P_{I}}-P_{R}\right) & ,|P|<P_{I}\end{cases}
$$

and Gibbs energy

$$
G(E, P)=\psi(P)-E P
$$

where $E$ and $P$ are the electric field and polarization and $P_{I}$ and $P_{R}$ denote the inflection point and polarization at which the minimum of $\psi$ occurs.

In the absence of thermal activation, minimization of $G$ for fixed field inputs yields the local polarization relation

$$
\bar{P}(E)=\frac{E}{\eta}+\delta P_{R}
$$

where the parameter $\delta$ has a value of 1 for positively oriented dipoles and -1 for negative orientations. To specify $\delta$ and hence $\bar{P}$ more specifically in terms of the initial dipole orientations and previous switches, we employ Preisach notation and take

$$
\left[\bar{P}\left(E ; E_{c}, \xi\right)\right](t)= \begin{cases}{\left[\bar{P}\left(E ; E_{c}, \xi\right)\right](0)} & , \tau(t)=\emptyset \\ \frac{E}{\eta}-P_{R} & , \tau(t) \neq \emptyset \text { and } E(\max \tau(t))=-E_{c} \\ \frac{E}{\eta}+P_{R} & , \tau(t) \neq \emptyset \text { and } E(\max \tau(t))=E_{c}\end{cases}
$$

Here

$$
\left[\bar{P}\left(E ; E_{c}, \xi\right)\right](0)= \begin{cases}\frac{E}{\eta}-P_{R} & , E(0) \leq-E_{c} \\ \xi & ,-E_{c}<E(0)<E_{c} \\ \frac{E}{\eta}+P_{R} & , E(0) \geq E_{c}\end{cases}
$$

denotes the initial dipole distribution and transition times are designated by

$$
\tau(t)=\left\{t \in\left(0, T_{f}\right] \mid E(t)=-E_{c} \text { or } E(t)=E_{c}\right\}
$$

where $T_{f}$ denotes the final time under consideration. The local coercive field

$$
E_{c}=\eta\left(P_{R}-P_{I}\right)
$$

quantifies the field at which the negative well ceases to exist and hence a dipole switch occurs. As shown in Figure 1, the kernel (3) or (4) quantifies the abrupt hysteresis transitions associated with homogeneous, single crystal compounds in the absence of thermal activation. 


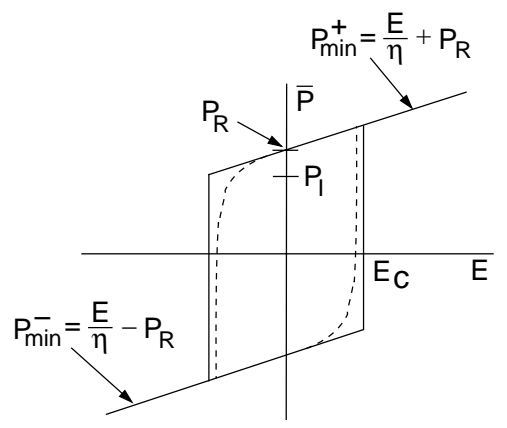

Figure 1: Local polarization $\bar{P}$ given by equation (6) with high thermal activation $(---)$ and limiting polarization $\bar{P}$ specified by (4) in the absence of thermal activation ( - ).

To incorporate thermal activation, the Gibbs energy $G$ and relative thermal energy $k T / V$ are balanced through the Boltzmann relation

$$
\mu(G)=C e^{-G V / k T} .
$$

Here $k$ is Boltzmann's constant, $V$ is a reference volume, $T$ is the temperature in degrees Kelvin, and $C$ is a constant chosen to ensure that integration over all admissible inputs yields a probability $\mu$ of unity. As detailed in [25], the kernel in this case is

$$
\bar{P}=x_{+}\left\langle P_{+}\right\rangle+x_{-}\left\langle P_{-}\right\rangle
$$

where $x_{+}$and $x_{-}$denote the fractions of dipoles having positive and negative orientations and $\left\langle P_{+}\right\rangle$ and $\left\langle P_{-}\right\rangle$denote the average expected polarizations associated with the two orientations. Since the expected polarization values are obtained by integrating the product $P \mu(G(P))$ over all admissible configurations, it follows that

$$
\left\langle P_{+}\right\rangle=\frac{\int_{P_{I}}^{\infty} P e^{-G(E, P, T) V / k T} d P}{\int_{P_{I}}^{\infty} e^{-G(E, P, T) V / k T} d P} \quad, \quad\left\langle P_{-}\right\rangle=\frac{\int_{-\infty}^{-P_{I}} P e^{-G(E, P) V / k T} d P}{\int_{-\infty}^{-P_{I}} e^{-G(E, P) V / k T} d P}
$$

The denominator results from the evaluation of the integration constant $C$ whereas it is illustrated in [25] that the use of the inflection points $\pm P_{I}$, to simplify evaluation of the integrals, can be justified though either asymptotic analysis or energy arguments.

Debye arguments yield the differential equations

$$
\begin{aligned}
& \dot{x}_{+}=-p_{+-} x_{+}+p_{-+} x_{-} \\
& \dot{x}_{-}=-p_{-+} x_{-}+p_{+-} x_{+}
\end{aligned}
$$

quantifying the evolution of the respective dipole fractions. For implementation, these relations can be simplified to the single differential equation

$$
\dot{x}_{+}=-p_{+-} x_{+}+p_{-+}\left(1-x_{+}\right)
$$

through the identity $x_{+}+x_{-}=1$. 
The likelihoods of switching from positive to negative orientations, or vice versa, are respectively quantified by

$$
p_{+-}=\frac{1}{\mathcal{T}(T)} \frac{\int_{P_{I}}^{P_{I}+\epsilon} e^{-G\left(E, P_{I}, T\right) V / k T} d P}{\int_{P_{I}}^{\infty} e^{-G(E, P, T) V / k T} d P} \quad, \quad p_{-+}=\frac{1}{\mathcal{T}(T)} \frac{\int_{-P_{I}-\epsilon}^{-P_{I}} e^{-G\left(E,-P_{I}, T\right) V / k T} d P}{\int_{-\infty}^{-P_{I}} e^{-G(E, P, T) V / k T} d P}
$$

where $\epsilon$ is taken to be a small positive constant. The relaxation term $\mathcal{T}$ quantifies the frequency at which jumps are attempted whereas the remainder of the definition characterizes the probability of achieving the energy required to exit respective potential wells. It is detailed in [25] that this probability increases when the relative thermal energy $k T / V$ approaches the Gibbs energy $G$.

Remark 1. It is proven in [17, 25] that the kernel $\bar{P}$ given by (6) converges to the piecewise linear kernel (3) or (4) in the limit $k T / V \rightarrow 0$ of negligible thermal activation. It also follows immediately that $\bar{P}$ given by (6) satisfies

$$
\begin{aligned}
& \bar{P} \leq P_{\min }^{+}=\frac{E}{\eta}+P_{R}, \text { positively oriented dipoles } \\
& \bar{P} \geq P_{\min }^{-}=\frac{E}{\eta}-P_{R}, \text { negatively oriented dipoles }
\end{aligned}
$$

as depicted in Figure 1. For all fields $E \in C[a, b]$ with $a, b$ finite, it thus follows that $\bar{P} \in L^{1}(a, b)$ and $\bar{P} \in L^{2}(a, b)$ for $\bar{P}$ given by (3), (4) or (6).

\subsubsection{Macroscopic Polarization Model}

To incorporate the effects of material nonhomogeneities, polycrystallinity, and variable effective fields $E_{e}=E+E_{I}$, where $E_{I}$ denotes interaction fields, we assume that $E_{I}$ and the local coercive field $E_{c}$, defined in (5), are manifestations of underlying distributions rather than constants. This yields the macroscopic model

$$
\begin{aligned}
{[P(E)](t) } & =\int_{0}^{\infty} \int_{-\infty}^{\infty} \nu_{1}\left(E_{c}\right) \nu_{2}\left(E_{I}\right)\left[\bar{P}\left(E+E_{I} ; E_{c}, \xi\right)\right](t) d E_{I} d E_{c} \\
& =\int_{0}^{\infty} \int_{-\infty}^{\infty} \nu\left(E_{c}, E_{I}\right)\left[\bar{P}\left(E+E_{I} ; E_{c}, \xi\right)\right](t) d E_{I} d E_{c}
\end{aligned}
$$

where $\nu_{1}$ and $\nu_{2}$ are densities associated with $E_{c}$ and $E_{I}$.

To accommodate physical criteria, we assume that $\nu_{1} \geq 0$ and $\nu_{2} \geq 0$ satisfy the conditions

$$
\begin{array}{ll}
\text { (i) } & \nu_{1}(x) \text { defined for } x>0, \\
\text { (ii) } & \nu_{2}(-x)=\nu_{2}(x) \\
\text { (iii) } & \left|\nu_{1}(x)\right| \leq c_{1} e^{-a_{1} x} \\
& \left|\nu_{2}(x)\right| \leq c_{2} e^{-a_{2}|x|}
\end{array}
$$

for positive $c_{1}, a_{1}, c_{2}, a_{2}$. The restricted domain in (i) reflects the fact that the coercive field $E_{c}$ is positive whereas the symmetry enforced in the interaction field through (ii) yields the symmetry observed in low-field Rayleigh loops. Hypothesis (iii) incorporates the physical observation that the 
coercive and interaction fields decay as a function of distance and guarantees that integration against the piecewise linear kernel yields finite polarization values.

Remark 2. It is illustrated in [17,20] that for certain operating regimes, reasonable accuracy can be obtained using a priori choices for $\nu_{1}$ and $\nu_{2}$. Motivated by densities employed in Preisach models for magnetic compounds, one such choice is

$$
\begin{aligned}
& \nu_{1}\left(E_{c}\right)=c_{1} e^{-\left[\ln \left(E_{c} / \bar{E}_{c}\right) / 2 c\right]^{2}} \\
& \nu_{2}\left(E_{I}\right)=c_{2} e^{-E_{I}^{2} / 2 b^{2}} .
\end{aligned}
$$

For general operating regimes, however, identification of general densities $\nu_{1}$ and $\nu_{2}$ is necessary to achieve accurate material characterization throughout both biased and unbiased operating regimes.

Remark 3. Formulation of the model in terms of the joint density $\nu\left(E_{c}, E_{I}\right)=\nu_{1}\left(E_{c}\right) \nu_{2}\left(E_{I}\right)$ increases the dimensionality of the parameter estimation problem but yields models that exhibit a linear dependence on parameters. This proves advantageous when constructing linear adaptive control techniques and for this reason, we focus heavily on this case in subsequent analysis.

\subsubsection{Discretized Polarization Model}

For implementation purposes, Gaussian or Newton-Cotes quadrature routines can be employed to approximate the integrals, thus yielding the system

$$
[P(E)](t)=\sum_{i=1}^{N_{i}} \sum_{j=1}^{N_{j}} \nu_{1}\left(E_{c_{i}}\right) \nu_{2}\left(, E_{I_{j}}\right)\left[\bar{P}\left(E_{I_{j}}+E ; E_{c_{i}}, \xi\right)\right](t) v_{i} w_{j}
$$

where $v_{i}$ and $w_{j}$ are the weights associated with the quadrature rules and $E_{c_{i}}, E_{I_{j}}$ are the abscissas. Highly efficient algorithms for implementing the approximate model (10) with general densities $\nu_{1}$ and $\nu_{2}$ are provided in $[17,25]$.

Formulation of the model in terms of the joint density $\nu=\nu_{1} \cdot \nu_{2}$ increases the dimensionality of the parameter estimation problem from $N_{i}+N_{j}$ to $N_{i} \cdot N_{j}$ but yields a system which depends linearly on the parameters $\left\{\nu\left(E_{c_{i}}, E_{I_{j}}\right)\right\}$. This permits implementation of linear least squares algorithms and linear adaptive identification and control techniques.

To formulate the discretized model (10) as a linear system in terms of $\nu=\nu_{1} \cdot \nu_{2}$, we define the $N_{i} \times N_{j}$ matrices $A(E)$ and $\Phi$ to have components

$$
\begin{aligned}
& {[A(E)]_{i j}=\left[\frac{E+E_{I_{j}}}{\eta}+P_{R} \delta\left(E ; E_{c_{i}}, E_{I_{j}}\right)\right] v_{i} w_{j}} \\
& {[Q]_{i j}=\nu\left(E_{c_{i}}, E_{I_{j}}\right) .}
\end{aligned}
$$

For $N=N_{i} \cdot N_{j}$, we define the $N \times 1$ vector $q$ and $1 \times N$ vector $a(E)$ by

$$
q=\operatorname{vec}(Q) \quad, \quad a(E)=[\operatorname{vec}(A(E))]^{T}
$$

where 'vec' denotes the vector concatenation of the respective matrices. The discretized polarization model (10) can then be formulated as the linear system

$$
P(E)=a(E) q .
$$

We note that $\eta$ is considered known and fixed in this formulation and is incorporated in $a(E)$. 


\subsection{Magnetization Model}

The magnetization model is analogous so we simply summarize it here. Details regarding its development can be found in $[18,19]$.

The hysteretic relation between the magnetic field $H$ and magnetization $M$ in ferromagnetic materials is characterized by the model

$$
[M(H)](t)=\int_{0}^{\infty} \int_{-\infty}^{\infty} \nu_{1}\left(H_{c}\right) \nu_{2}\left(H_{I}\right)\left[\bar{M}\left(H+H_{I} ; H_{c}, \xi\right)\right](t) d H_{I} d H_{c}
$$

In the absence of relaxation processes, the kernel $\bar{M}$ has the form

$$
\bar{M}=\frac{H}{\eta}+\delta M_{R}
$$

whereas it is given by

$$
\bar{M}=x_{+}\left\langle M_{+}\right\rangle+x_{-}\left\langle M_{-}\right\rangle
$$

when the Gibbs and relative thermal energies are balanced to incorporate thermal relaxation processes. The dipole fractions $x_{+}, x_{-}$, average magnetizations $\left\langle M_{+}\right\rangle,\left\langle M_{-}\right\rangle$, local remanence magnetization $M_{R}$, and switching parameter $\delta$ are defined in a manner analogous to their polarization counterparts as detailed in Section 2.1. As with the polarization model, the goal in the parameter identification problem is to estimate $\nu$ given data measurements $\left\{\widehat{H}_{k}, \widehat{M}_{k}\right\}, k=1, \ldots, N_{d}$.

\subsection{Shape Memory Alloy Model}

The relation between input stresses $\sigma$ and strains $\varepsilon$ generated in shape memory alloy compounds as they undergo martensitic phase transformations is quantified by the integral relation

$$
[\varepsilon(\sigma, T)](t)=\int_{0}^{\infty} \int_{-\infty}^{\infty} \nu_{1}\left(\sigma_{R}\right) \nu_{2}\left(\sigma_{I}\right)\left[\bar{\varepsilon}\left(\sigma+\sigma_{I}, T ; \sigma_{R}, \xi\right)\right](t) d \sigma_{I} d \sigma_{R}
$$

which has the same general form as the polarization model (7) and magnetization model (12). For regimes in which thermal activation or relaxation mechanisms are significant, the kernel $\bar{\varepsilon}$ is given by

$$
\bar{\varepsilon}=x_{-}\left\langle\varepsilon_{-}\right\rangle+x_{+}\left\langle\varepsilon_{+}\right\rangle+x_{A}\left\langle\varepsilon_{A}\right\rangle
$$

where $x_{-}, x_{+}$and $x_{A}$ respectively denote the volume fraction of martensite minus, martensite plus, and austenite layers in 1-D SMA compounds and $\left\langle\varepsilon_{-}\right\rangle,\left\langle\varepsilon_{+}\right\rangle$and $\left\langle\varepsilon_{A}\right\rangle$ are the average strains associated with layers. Details regarding the construction of thermally active and inactive SMA models can be found in $[9,10,15,17]$.

\section{Compactness of the Polarization Operator}

The behavior of the inverse problems associated with estimating the densities $\nu_{1}$ and $\nu_{2}$, or the joint density $\nu=\nu_{1} \cdot \nu_{2}$, is intimately related to properties of the integral operators resulting from the models (7), (12) and (13). In this section, we establish that the operators are compact; in Section 4, we use this fact to establish the ill-posedness of the inverse problem associated with parameter estimation. To simplify the discussion, we focus solely on the polarization model in subsequent sections. Due to the unified nature of the characterization framework, however, the results also apply to the magnetization and SMA models. 
We focus on the case $\nu=\nu_{1} \cdot \nu_{2}$, which yields a linearly parameterized problem but comes at the cost of increased dimensionality. The kernel $\bar{P}$ in (7) can be taken as either the piecewise linear relation (3) or (4), that arises when thermal activation is negligible, or the kernel (6) which incorporates thermal processes. The important observation in both cases is the property that for finite input fields $E \in C[a, b]$, the kernel satisfies $\bar{P} \in L^{1}(a, b)$ and $\bar{P} \in L^{2}(a, b)$ as noted in Remark 1 .

It was noted in Section 1 that the homogenized energy framework provides an energy basis for certain extended Preisach models and hence one would expect similar compactness results for the two theories. The reader is referred to Iyer and Shirley [7] for theory establishing the compactness of the classical Preisach operator.

By invoking the physical decay criteria (8), the polarization model (7) can be approximated to arbitrary accuracy by the relation

$$
[P(E)](t)=\iint_{\Omega_{2}} \nu\left(E_{c}, E_{I}\right)\left[\bar{P}\left(E+E_{I} ; E_{c}, \xi\right)\right](t) d E_{I} d E_{c}
$$

on the compact domain

$$
\Omega_{2}=\left\{\left(E_{c}, E_{I}\right) \in \mathbb{R}_{+} \times \mathbb{R} \mid \nu\left(E_{c}, E_{I}\right) \geq \epsilon\right\} .
$$

Furthermore, we let the minimum and maximum admissible input fields be denoted $E_{\min }$ and $E_{\max }$ and define

$$
\Omega_{1}=\left[E_{\min }, E_{\max }\right] .
$$

We consider parameters $q=\nu$ in the parameter space

$$
\mathcal{Q}=L^{2}\left(\Omega_{2}\right)
$$

and define the observation operator $\mathcal{C} P=P(E)$ on the observation space

$$
\mathcal{Y}=L^{2}\left(P_{\min }, P_{\max }\right)
$$

The polarization model (7) can then be formulated as

$$
y(E)=\mathcal{K} q(E)
$$

where

$$
E \in C\left[\Omega_{1}\right] \subset L^{2}\left(\Omega_{1}\right)
$$

and the parameter-to-observation operator $\mathcal{K}$ is defined by

$$
\mathcal{K} q=\mathcal{C} \iint_{\Omega_{2}} k\left(\cdot+E_{I}, E_{c}\right) q\left(E_{c}, E_{I}\right) d E_{I} d E_{c} .
$$

From the property that $\bar{P}$ given by (4) or (6) satisfies $\bar{P} \in L^{1}\left(\Omega_{1}\right), \bar{P} \in L^{2}\left(\Omega_{1}\right)$, as noted in Remark 1 , it follows that $k \in L^{1}(\Omega)$ and $k \in L^{2}(\Omega)$ where

$$
\Omega=\Omega_{1} \times \Omega_{2} .
$$

The property that $k \in L^{1}(\Omega)$ is typical for convolution operators whereas $k \in L^{2}(\Omega)$ facilitates construction of a generalized Fourier basis for the operator. We employ this latter property to establish that $\mathcal{K}$ is compact for the given choice of spaces. As a prelude, we state the following theorem which is Theorem 5.24.8 from [13]. 
Theorem 1. Let $X$ and $Y$ be Banach spaces and let $\mathcal{K}_{N}: X \rightarrow Y, N=1,2, \ldots$, be a sequence of compact linear operators converging to a bounded linear operator $\mathcal{K}: X \rightarrow Y$; that is, $\left\|\mathcal{K}_{N}-\mathcal{K}\right\| \rightarrow 0$ as $N \rightarrow \infty$. Then $\mathcal{K}$ is a compact linear operator.

Remark 4. Consider the parameter space $\mathcal{Q}$ and observation space $\mathcal{Y}$ defined in (15) and (16). The integral operator given by (17) is then a compact operator. We establish this by demonstrating that $\mathcal{K}$ is the limit of a sequence of finite rank operators followed by the use of Theorem 1.

We first construct an orthonormal basis $\left\{\phi_{i}\right\}$ for $L^{2}(\Omega)$. It is illustrated in [13] that

$$
\varphi_{\ell}(s)=\frac{1}{\sqrt{E_{\text {max }}-E_{\min }}} \exp \left[2 \pi i \ell \cdot \frac{s-E_{\min }}{\ell-E_{\min }}\right], \ell=0, \pm 1, \pm 2, \cdots
$$

forms an orthonormal basis for $L^{2}\left(\Omega_{1}\right)$. With an analogous basis definition for $L^{2}\left(\Omega_{2}\right)$, it follows that an orthonormal basis for $L^{2}(\Omega)$ is

$$
\phi_{\ell m}(s, t, v)=\varphi_{\ell}(s) \overline{\varphi_{m}(t, v)}
$$

which we re-index as $\left\{\phi_{i}\right\}$.

It follows that every $f \in L^{2}(\Omega)$ has the generalized Fourier series representation

$$
f=\sum_{i}\left\langle f, \phi_{i}\right\rangle \phi_{i}
$$

where $\langle\cdot, \cdot\rangle$ denotes the usual $L^{2}$ inner product. The norm representation

$$
\|f\|^{2}=\sum_{i}\left|\left\langle f, \phi_{i}\right\rangle\right|^{2}
$$

follows from Plancheral's theorem. Moreover, we can represent $\mathcal{K}$ and approximating finite-rank operators $\mathcal{K}_{N}$ by

$$
\begin{gathered}
\mathcal{K} f=\sum_{i}\left\langle f, \phi_{i}\right\rangle \psi_{i} \\
\mathcal{K}_{N} f=\sum_{i=1}^{N}\left\langle f, \phi_{i}\right\rangle \psi_{i}
\end{gathered}
$$

where $\psi_{i} \equiv \mathcal{K} \phi_{i}$.

To establish the convergence $\mathcal{K} \rightarrow \mathcal{K}_{N}$, we note that

$$
\begin{aligned}
\left\|\mathcal{K} f-\mathcal{K}_{N} f\right\| & =\left\|\sum_{i \geq N+1}\left\langle f, \phi_{i}\right\rangle \psi_{i}\right\| \\
& \leq \sum_{i \geq N+1}\left|\left\langle f, \phi_{i}\right\rangle\right|\left\|\psi_{i}\right\| \\
& \leq\left[\sum_{i \geq N+1}\left|\left\langle f, \phi_{i}\right\rangle\right|^{2}\right]^{1 / 2}\left[\sum_{i \geq N+1}\left\|\psi_{i}\right\|^{2}\right]^{1 / 2} \\
& \leq\|f\|\left[\sum_{i \geq N+1}\left\|\psi_{i}\right\|^{2}\right]^{1 / 2}
\end{aligned}
$$


where the third inequality follows from the Schwartz inequality. Furthermore, we observe that

$$
\begin{aligned}
\sum_{i}\left\|\psi_{i}\right\|^{2} & =\sum_{i}\left[\int_{\Omega_{1}}\left|\mathcal{K} \phi_{i}(E)\right|^{2} d E\right] \\
& =\sum_{i} \int_{\Omega_{1}}\left|\iint_{\Omega_{2}} k\left(E+E_{I}, E_{c}\right) \phi_{i}\left(E_{c}, E_{I}\right) d E_{I} d E_{c}\right|^{2} d E \\
& =\int_{\Omega_{1}}\left[\sum_{i}\left|\iint_{\Omega_{2}} k\left(E+E_{I}, E_{c}\right) \phi_{i}\left(E_{c}, E_{I}\right) d E_{I} d E_{c}\right|^{2}\right] d E \\
& =\int_{\Omega_{1}}\left[\iint_{\Omega_{2}}\left|k\left(E+E_{I}, E_{c}\right)\right|^{2} d E_{I} d E_{c}\right] d E<\infty .
\end{aligned}
$$

The last step follows from Plancheral's theorem. Convergence of the series $\sum_{i}\left\|\psi_{i}\right\|^{2}$ implies that $\sum_{i \geq N+1}\left\|\psi_{i}\right\|^{2} \rightarrow 0$ as $N \rightarrow \infty$. Thus for $\varepsilon>0$, there exists $N_{\varepsilon}$ such that for $N>N_{\varepsilon}$,

$$
\left\|\mathcal{K}-\mathcal{K}_{N}\right\|=\sup _{f \neq 0} \frac{\left\|\mathcal{K} f-\mathcal{K}_{N} f\right\|}{\|f\|}<\varepsilon
$$

which establishes that

$$
\lim _{N \rightarrow \infty}\left\|\mathcal{K}-\mathcal{K}_{N}\right\|=0
$$

Since the range of $\mathcal{K}_{N}$ is finite, it follows that $\mathcal{K}_{N}$ is a compact operator. The compactness of $\mathcal{K}$ follows from Theorem 1 since it is the norm limit of a sequence of compact operators.

\section{Parameter Identification Problem}

We focus on the problem of identifying the joint density $\nu$ in the polarization model (7) or density values $\left\{\nu\left(E_{c_{i}}, E_{I_{j}}\right)\right\}$ in the discretized problem (10) due to the fact that these formulations yield the linear parameterization necessary for linear adaptive identification or control techniques. The formulation of parameter estimation problems associated with the identification of independent densities $\nu_{1}$ and $\nu_{2}$ or parameters arising in functional representations for $\nu_{1}$ and $\nu_{2}-$ e.g., $C=c_{1} \cdot c_{2}, \bar{E}_{c}, c, b$ in (9) - is analogous but requires nonlinear parameterizations in the operator and matrix formulations.

For the operator $\mathcal{K}$ defined in (17), data $\widehat{P}$ corresponding to input fields $\widehat{E} \in L^{2}\left(E_{\min }, E_{\max }\right)$, and parameter space $\mathcal{Q}=L^{2}\left(\Omega_{2}\right)$, the parameter estimation problem can be formulated as follows: find $q \in \mathcal{Q}$ so that

$$
\mathcal{K} q=\widehat{P} .
$$

We note that (18) has a classical solution if and only if $\widehat{P} \in \mathcal{R}(\mathcal{K})$, where $\mathcal{R}(\mathcal{K})$ denotes the range of $\mathcal{K}$, which, in general, will not be true. Instead it is more reasonable to consider the least squares problem

$$
\min _{q \in \mathcal{Q}} T(q), T(q)=\frac{1}{2}\|\mathcal{K} q-\widehat{P}\|_{\mathcal{Y}}^{2} .
$$

However, because $\mathcal{K}$ is compact with infinite dimensional range, the Moore-Penrose inverse $\mathcal{K}^{\dagger}$ is discontinuous so that even (19) is ill-posed - see [4]. This motivates consideration of the augmented functional

$$
T_{\alpha}(q)=\frac{1}{2}\|\mathcal{K} q-\widehat{P}\|_{\mathcal{Y}}^{2}+\alpha \mathcal{J}(q)
$$


and the regularized least squares minimization problem

$$
\min _{q \in \mathcal{Q}} T_{\alpha}(q)
$$

The regularization parameter $\alpha>0$ controls the tradeoff between goodness of fit to the data and stability whereas the penalty functional $\mathcal{J}$ provides stability and allows the inclusion of a priori information regarding the parameter $q$. One choice for $\mathcal{J}$ is the Tikhonov functional which we illustrate in the context of the discretized problem.

To formulate the finite-dimensional parameter estimation problem, we modify the linearly parameterized system (11) to reflect measured data. We define the $N_{i} \times N_{j}$ matrices

$$
\begin{aligned}
& {\left[A_{k}\right]_{i j}=\left[\frac{\widehat{E}_{k}+E_{I_{j}}}{\eta}+P_{R} \delta\left(\widehat{E}_{k} ; E_{c_{i}}, E_{I_{j}}\right)\right] v_{i} w_{j}} \\
& {[Q]_{i j}=\nu\left(E_{c_{i}}, E_{I_{j}}\right)}
\end{aligned}
$$

and vector concatenations

$$
q=\operatorname{vec}(Q) \quad, \quad a_{k}=\left[\operatorname{vec}\left(A_{k}\right)\right]^{T}
$$

so that $q$ and $a_{k}$ are respectively $1 \times N$ and $N \times 1$ where $N=N_{i} \cdot N_{j}$. Additionally, the $N_{d} \times 1$ vectors $\mathcal{P}$ and $\widehat{\mathcal{P}}$ are defined componentwise by

$$
[\mathcal{P}]_{k}=P\left(\widehat{E}_{k} ; q\right) \quad, \quad[\widehat{\mathcal{P}}]_{k}=\widehat{P}_{k}
$$

and the $N_{d} \times N$ matrix $A$ is defined row-wise by

$$
[A]_{k}=a_{k} .
$$

The discretized polarization model (7) can then be formulated as the linearly parameterized system

$$
\mathcal{P}\left(\widehat{E}_{k}\right)=A q
$$

— see $[17,25]$ for highly efficient implementation algorithms.

The unregularized least squares problem used to estimate $q=\nu \in Q=\mathbb{R}^{N_{i} \cdot N_{j}}$ given measurements $\left\{\left(\widehat{E}_{k}, \widehat{P}_{k}\right)\right\}, k=1, \ldots, N_{d}$ is the following:

$$
\begin{aligned}
& \min _{q \in Q} T(q), T(q)=\frac{1}{2}\|A q-\widehat{\mathcal{P}}\|^{2} \\
& \text { subject to } q_{i} \geq 0, j=1, \ldots, N .
\end{aligned}
$$

Here $\|\cdot\|$ denotes the Euclidean norm in $\mathbb{R}^{N}$. To incorporate Tikhonov regularization, we consider the minimization problem

$$
\begin{aligned}
& \min _{q \in Q} T_{\alpha}(q), T(q)=\frac{1}{2}\left\|A q-\widehat{\mathcal{P}}_{k}\right\|^{2}+\frac{\alpha}{2}\|q\|^{2} \\
& \text { subject to } q_{i} \geq 0, j=1, \ldots, N .
\end{aligned}
$$

Techniques for choosing $\alpha$ to avoid oversmoothing solutions as well as a solution algorithm for (24) can be found in Vogel [27].

We do not consider the convergence of approximate parameters as discretization levels are increased but instead let the infinite-dimensional analysis motivate potential sources of ill-posedness in the discrete least squares formulations. The effects of ill-posedness are demonstrated in the context of material characterization using experimental data. 


\section{Material Characterization}

To illustrate attributes of the least squares parameter estimation formulations (23) and (24) for estimating the $N=N_{i} \cdot N_{j}$ parameters $\left\{\nu\left(E_{c_{i}}, E_{I_{j}}\right)\right\}$, we consider the characterization of the ferroelectric compound PZT5H using experimental data which includes both an unbiased major loop and multiple biased minor loops. Because data was collected at $0.2 \mathrm{~Hz}$, relaxation effects were negligible so we employed the piecewise linear kernel (3) or (4) in the discretized model (10).

The unregularized model fits obtained with the discretization limits $N_{i}=N_{j}=24(N=576)$ and $N_{i}=N_{j}=48(N=2304)$ using data from all seven hysteresis loops are plotted in Figure 2 whereas those obtained using the same quadrature limits in the regularized functional are given in Figure 3. Without regularization, the ill-posedness associated with inversion of the discretized compact operator yields increasingly inaccurate model predictions as quadrature limits are increased. Regularization through the inclusion of the penalty term $\frac{\alpha}{2}\|q\|^{2}$ stabilizes the pseudoinverse by shifting singular values away from the origin thus yielding the highly accurate fit observed in Figure 3. The regularization parameter $\alpha=5 \times 10^{20}$ used to obtain the modeled behavior in Figure 3 was computed using a variation of the unbiased predictive risk estimator (UPRE) method discussed in Vogel [27]. Smaller and larger values of $\alpha$ yielded larger residuals and increasingly inaccurate modeled behavior - e.g., the fit in Figure 2 corresponds to $\alpha=0$.

Additional examples illustrating the performance of the model with a priori functional choices for $\nu_{1}$ and $\nu_{2}$ can be found in [20] where it is demonstrated that general densities are required to obtain accurate characterization through the full hierarchy of drive conditions.

\section{Concluding Remarks}

This paper addresses theoretical and implementation issues associated with the construction of models used to characterize hysteresis and constitutive nonlinearities inherent to ferroelectric, ferromagnetic and ferroelastic materials. This unified characterization framework employs energy analysis to construct polarization, magnetization and strain kernels at the lattice level. To incorporate the effects of material nonhomogeneities, polycrystallinity, and variable effective fields, certain parameters are assumed to be manifestations of underlying distributions rather than constants. Stochastic homogenization in this manner yields integral formulations having known kernels and unknown den-

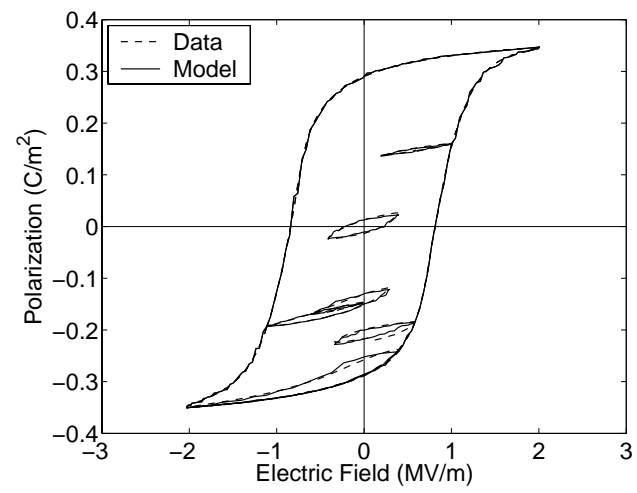

(a)

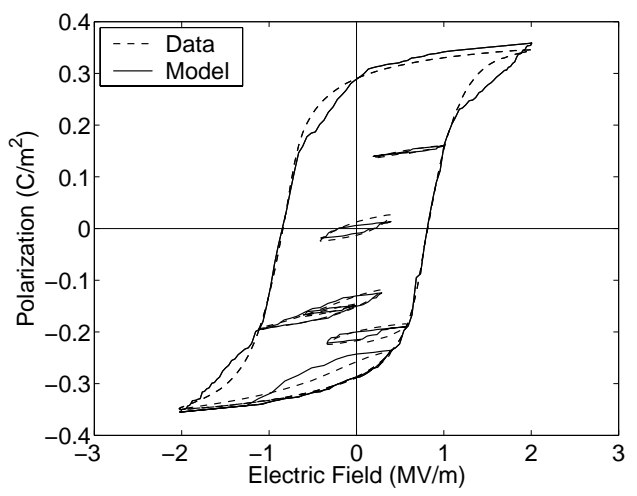

(b)

Figure 2: PZT5H data and model fit with general product density $\nu$ estimated using the unregularized functional (23) with data from all 7 loops. (a) $N_{i}=N_{j}=24(N=576)$, and (b) $N_{i}=N_{j}=48$ $(N=2304)$. 


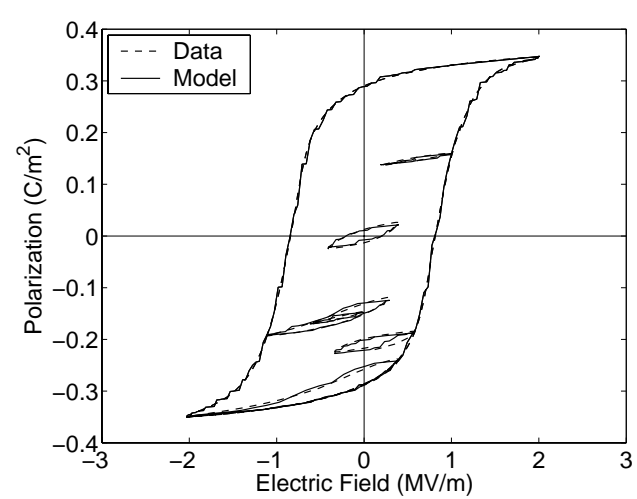

(a)

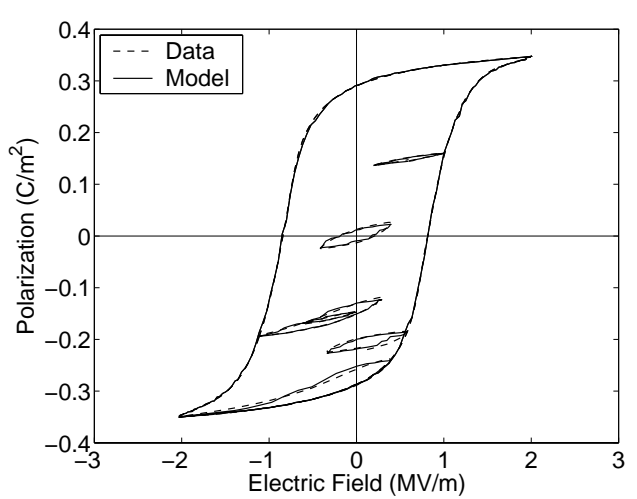

(b)

Figure 3: PZT5H data and model fit with general product density $\nu$ estimated using the regularized Tikhonov functional (24) with data from all 7 loops. (a) $N_{i}=N_{j}=24$ ( $N=576$ ), and (b) $N_{i}=N_{j}=48(N=2304)$.

sities which must be estimated for a given material of transducer configuration. Estimation of either these general densities, or parameters in assumed functional forms for the densities, comprises the parameter estimation problem under consideration in this paper.

It is noted that in this homogenized energy framework, one has the option of estimating general constituent densities $\nu_{1}$ and $\nu_{2}$, the joint density $\nu=\nu_{1} \cdot \nu_{2}$, or functional forms of $\nu_{1}$ and $\nu_{2}$ having a small number of parameters. It is illustrated in [20] that the third option leads to a small number of parameter to be identified (e.g., 5 to 6 ) but yields models having limited accuracy for general operating regimes. Consideration of the joint density $\nu$ increases the dimensionality of the parameter estimation problem but yields a linear parameterization as required for linear adaptive identification and control techniques. For this reason, we focused on this formulation throughout the development.

It is demonstrated that the integral operators associated with the model are compact and hence the inverse problem is ill-posed. This motivates consideration of Tikhonov regularization to stabilize the pseudoinverse by shifting singular values away from the origin. It is shown in the context of characterizing PZT5H material behavior that increasingly inaccurate model fits are obtained as discretization levels are increased in the absence of regularization whereas the deleterious effects of ill-posedness are avoided when suitable regularization is incorporated.

From a practical perspective, the decrease in regularity and accuracy due to ill-posedness can be mitigated by employing small to moderate discretization limits - this forms the basis of regularization by coarsening. Whereas convergence issues are not addressed by this latter approach, it yields models which can facilitate control design.

\section{Acknowledgements}

This research was supported through the NSF grants CMS-009764 and CMS-0201560 and the Air Force Office of Scientific Research under the grant AFOSR-F49620-01-1-0107. 


\section{References}

Note: Center for Research in Scientific Computation Technical Reports can be accessed at the web site http://www.ncsu.edu/crsc/reports.html.

[1] E. Della Torre, Magnetic Hysteresis, IEEE Press, New York, 1999.

[2] W.S. Galinaitis and R.C. Rogers, "Compensation for hysteresis using bivariate Preisach models," Smart Structures and Materials 1997, Proceedings of the SPIE, Volume 3039, pp. 538-547, 1997.

[3] P. Ge and M. Jouaneh, "Modeling hysteresis in piezoceramic actuators," Precision Engineering, 17, pp. 211-221, 1995.

[4] C.W. Groetsch, The Theory of Tikhonov Regularization for Fredholm Equations of the First Kind, Pitman, Boston, 1984.

[5] R.V. Iyer, "Recursive estimation of the Preisach density function for a smart actuator," Smart Structures and Materials 2004, Proceedings of the SPIE, Volume 5383, pp. 202-210, 2004.

[6] R.V. Iyer and P.S. Krishnaprasad, "On a low-dimensional model for ferromagnetism," Nonlinear Analysis: Theory, Methods and Application, to appear.

[7] R.V. Iyer and M.E. Shirley, "Hysteresis parameter identification with limited experimental data," IEEE Transactions on Magnetics, 40(5), pp. 3227-3239, 2004.

[8] J.E. Massad and R.C. Smith, "A domain wall model for hysteresis in ferroelastic materials," Journal of Intelligent Material Systems and Structures, 14(7), pp. 455-471, 2003.

[9] J.E. Massad and R.C. Smith, "A homogenized free energy model for hysteresis in thin-film shape memory alloys," CRSC Technical Report CRSC-TR04-26; International Journal on the Science and Technology of Condensed Matter Films, to appear.

[10] J.E. Massad, R.C. Smith and G.P. Carman, "A free energy model for thin-film shape memory alloys," Smart Structures and Materials 2003, Proceedings of the SPIE, Volume 5049, pp. 13-23, 2003.

[11] I.D. Mayergoyz, "Mathematical models of hysteresis," IEEE Transactions on Magnetics, 22(5), pp. 603-608, 1986.

[12] I.D. Mayergoyz, Mathematical Models of Hysteresis, Springer-Verlag, New York, 1991.

[13] A.W. Naylor and G.R. Sell, Linear Operator Theory in Engineering and Science, SpringerVerlag, New York, 2000.

[14] D.C. Jiles and D.L. Atherton, "Theory of ferromagnetic hysteresis," Magnetism and Magnetic Materials, 61, pp. 48-60, 1986.

[15] S. Seelecke and I. Müller, "Shape memory alloy actuators in smart structures - Modeling and simulation," ASME Applied Mechanics Reviews, 57(1), pp. 23-46, 2004.

[16] M.E. Shirley and R. Venkataraman, "On the identification of Preisach measures," Smart Structures and Materials 2003, Proceedings of the SPIE, Volume 5049, pp. 326-336, 2003.

[17] R.C. Smith, Smart Material Systems: Model Development, SIAM, Philadelphia, PA, 2005. 
[18] R.C. Smith and M.J. Dapino, "A homogenized energy theory for ferromagnetic hysteresis," IEEE Transactions on Magnetics, submitted.

[19] R.C. Smith, M.J. Dapino and S. Seelecke, "A free energy model for hysteresis in magnetostrictive transducers," Journal of Applied Physics, 93(1), pp. 458-466, 2003.

[20] R.C. Smith, A. Hatch, B. Mukherjee and S. Liu, "A homogenized energy model for hysteresis in ferroelectric materials: General density formulation," CRSC Technical Report CRSC-TR04-23; Journal of Intelligent Material Systems and Structures, to appear.

[21] R.C. Smith and C.L. Hom, "Domain wall theory for ferroelectric hysteresis," Journal of Intelligent Material Systems and Structures, 10(3), pp. 195-213, 1999.

[22] R.C. Smith and J.E. Massad, "A unified methodology for modeling hysteresis in ferroic materials," Proceedings of the 2001 ASME Design Engineering Technical Conferences and Computers and Information in Engineering Conference, Vol 6, Pt B, pp. 1389-1398, 2001.

[23] R.C. Smith and S. Seelecke, "An energy formulation for Preisach models," Smart Structures and Materials 2002, Proceedings of the SPIE, Volume 4693, pp. 173-182, 2002.

[24] R.C. Smith, S. Seelecke, M.J. Dapino and Z. Ounaies, "A unified model for hysteresis in ferroic materials," Smart Structures and Materials 2003, Proceedings of the SPIE, Volume 5049, pp. 8899, 2003.

[25] R.C. Smith, S. Seelecke, Z. Ounaies and J. Smith, "A free energy model for hysteresis in ferroelectric materials," Journal of Intelligent Material Systems and Structures, 14(11), pp. 719-739, 2003.

[26] X. Tan, R. Venkataraman and P.S. Krishnaprasad, "Control of hysteresis: Theory and experimental results," Smart Structures and Materials 2001, Proceedings of the SPIE, Volume 4326, pp. 101-112, 2001.

[27] C.R. Vogel, Computational Methods for Inverse Problems, SIAM, Philadelphia, PA, 2002. 